Recherches en didactique des langues et des cultures

Les cahiers de l'Acedle

$15-2 \mid 2018$

Quelles médiations en didactique des langues et des cultures?

\title{
Migrations et médiations des langues et des hommes : quel rôle pour la didactique des langues?
}

\section{Emmanuelle Le Pichon-Vorstman}

\section{OpenEdition}

\section{Journals}

Édition électronique

URL : http://journals.openedition.org/rdlc/2985

DOI : $10.4000 /$ rdlc. 2985

ISSN : 1958-5772

Éditeur

ACEDLE

Référence électronique

Emmanuelle Le Pichon-Vorstman, « Migrations et médiations des langues et des hommes : quel rôle pour la didactique des langues? », Recherches en didactique des langues et des cultures [En ligne],

15-2 | 2018, mis en ligne le 02 juin 2018, consulté le 21 avril 2019. URL : http:// journals.openedition.org/rdlc/2985; DOI : 10.4000/rdlc.2985

Ce document a été généré automatiquement le 21 avril 2019

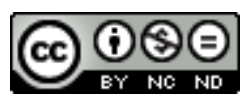

Recherches en didactique des langues et des cultures is licensed under a Creative Commons AttributionNonCommercial-NoDerivatives 4.0 International License 


\title{
Migrations et médiations des langues et des hommes : quel rôle pour la didactique des langues?
}

\author{
Emmanuelle Le Pichon-Vorstman
}

\section{Introduction : Evolution des modes de migration}

1 Au cours des dernières décennies, la mobilité des individus en Europe et dans le monde a augmenté à une vitesse étonnante (Union Européenne, 2012). Cette augmentation de la mobilité a évidemment des conséquences sur la société, en particulier, des conséquences linguistiques et culturelles. Le concept de diversité est apparu vers la fin des années 2000 et son usage s'est intensifié depuis 2010. Au sein de l'Union Européenne et au-delà, le concept fait rage. Mais qu'est-ce que la diversité ? Dans le contexte de cet article, le concept de diversité est lié à l'accroissement de la mobilité des personnes en Europe et hors d'Europe (Union Européenne, 2012). Ce phénomène a introduit un degré élevé d'hétérogénéité dans les classes, non seulement en termes de contexte linguistique et culturel, mais aussi en termes d'expériences académiques. En outre, il a diminué la stabilité des populations scolaires. En effet, de plus en plus d'apprenants passent d'un système à un autre à l'intérieur et à l'extérieur des frontières nationales. Selon Vertovec (2007), certaines parties de l'Europe peuvent même être qualifiées d'hyper-diverses puisqu'elles se distinguent " par un jeu dynamique de variables au milieu d'un nombre accru de migrants d'origines multiples, nouvelles, dispersées, transnationalement liées, differenciées socioéconomiquement et légalement stratifiées, arrivés au cours de la dernière décennie " (Vertovec, 2007: 1024). Ces nouveaux modèles de diversité posent de nouveaux défis à l'institution scolaire. De plus en plus, nous prenons conscience du fait que la diversité ne peut pas être comprise comme une fatalité à assumer mais qu'elle peut être mise à profit, adoptée ou même, qu'elle doit être embrassée si l'on veut pouvoir en tirer profit. Quelle médiation mettons-nous en place pour vivre cette diversité de manière harmonieuse ? La diversité est un enrichissement pour l'institution scolaire en général: elle implique l'accueil d'apprenants aux compétences linguistiques variées, l'ouverture vers des habitudes 
culturelles, des manières de penser et de vivre différentes. Parallèlement, elle implique une proportion croissante d'élèves qui sont amenés à communiquer en plusieurs langues : soit parce qu'ils évoluent d'un pays à l'autre, soit parce que leur(s) langue(s) maternelle(s) ne correspondent pas aux langues de l'école ; autant de cas de figure différents qu'il y a d'apprenants. Ensemble, ces phénomènes d'accroissement de la mobilité et de la diversité modifient progressivement le contexte des expériences linguistiques et culturelles des jeunes apprenants dans les systèmes scolaires européens (Blommaert, 2010).

2 Une étudiante de français langue étrangère, inscrite en master d'interculturel et spécialisée dans les problématiques qui entourent le plurilinguisme, s'est récemment méprise sur la terminologie, employant la formule « enfants migrateurs » au lieu de «migrants ». Lors d'une conférence pour des enseignants, l'une des questions posées par les organisateurs était: «Connaissez-vous des migrants?» Si la question est choquante, elle est intéressante à plus d'un titre : non seulement elle sous-entend l'étrangeté de l'individu en situation de migration mais on s'aperçoit aussi que le mot migrant implique un élément statique, contrairement au migrateur qui contient une idée mobilité continue. Qui sont ces apprenants ? D'où viennent-ils? Quel est le rôle que doit jouer la didactique des langues dans la médiation des langues et des cultures dans ces nouveaux environnements éducationnels? Mais aussi, jusqu'où sommes-nous prêts à aller, nous, enseignants et chercheurs? Où se situent nos propres résistances à la médiation en didactique des langues et des cultures? Qu'en pensent les apprenants? Quelles leçons en tirer pour l'avenir?

\section{Nouvelles caractéristiques migratoires}

\section{Une mobilité accrue}

3 Les caractéristiques concernant l'afflux récent d'apprenants nouvellement arrivés dans nos systèmes scolaires diffèrent à plusieurs titres des expériences antérieures. D'abord, le nombre d'arrivées sur les dix dernières années est sans précédent. Ensuite, les pays d'origine sont beaucoup plus variés qu'il y a vingt ans (Le Pichon- Vorstman, van Erning, Baauw, 2016). Sur 52 apprenants d'une école primaire d'une grande ville des Pays-Bas, nous recensions en 2014, 27 lieux de provenances différents. De plus, les pays de naissance des parents divergeaient souvent de ceux de leurs enfants, l'enfant pouvant être né dans un camp de réfugiés dans un pays plus ou moins éloigné du ou des pays d'origine de ses parents. La prise en compte des nationalités augmente encore la complexité de la tâche. C'est ainsi qu'une jeune fille à la nationalité américaine, était en réalité née à New York, de parents nés en Pologne, et de nationalité polonaise. Ces divergences témoignent déjà d'un parcours de mobilité important: mobilités réelles, c'est-à-dire accompagnées d'un déplacement géographique, mais aussi mouvances linguistiques et culturelles entrainées par ces divergences sans forcément que l'apprenant ait lui-même vécu cette mobilité parfois antérieure à sa naissance. Mais cette mobilité ne s'arrête pas là.

Des 52 enfants nouvellement arrivés rencontrés en avril 2014, tous scolarisés dans le même établissement primaire dans une grande ville des Pays-Bas, il n'en restait que 41 en juin de la même année (Le Pichon- Vorstman \& Baauw, 2014). Combien d'écoles primaires perdent presque vingt pourcents de leur population entre les mois d'avril et de juin d'une même année scolaire ? Pour illustrer cette mobilité post-migratoire, deux des élèves en provenance d'Afghanistan et qui avaient intégrées l'école néerlandaise quatre années 
auparavant, étaient partie pour l'Angleterre. Cela signifie que les Pays-Bas n'ont été qu'une étape de quatre ans dans la vie de ces enfants, étape pendant laquelle elles ont passé un an à apprendre le néerlandais et à apprendre à apprendre aux Pays-Bas, suivi de deux années à l'école primaire du quartier pour ensuite rejoindre l'Angleterre où il est probable que les autorités locales aient mis le même type de procédure en place. Cela nous interroge sur l'éthique qui sous-tend ces classes d'adaptation linguistique. En déplacement en Suède, j'ai eu l'occasion de parler en néerlandais à des parents érythréens qui venaient de passer dix années aux Pays-Bas avant d'émigrer vers la Suède. Un directeur d'école islandais exprimait récemment son désarroi nous demandant de le conseiller : que devait-il faire avec les enfants bulgares qui arrivent en Islande puisqu'ils n'ont aucune chance d'obtenir un permis de séjour mais que la procédure peut durer jusqu'à deux ans ? Faut-il intégrer ces enfants à l'école du village sachant qu'ils devront fournir un effort langagier et culturel important sans espoir de rester ou faut-il engager des enseignants bulgares en prenant le risque de priver ces enfants de contacts avec les enfants sur place? Dans quelle(s) mesure(s) le système scolaire est-il (in)apte à inclure les élèves internationaux en mouvement? Faut-il dire que ces enfants ne sont pas adaptés au système ? N'est-ce pas plutôt le système scolaire qui n'est pas adapté aux enfants?

5 En ce qui concerne les Pays-Bas au moins, les pays et lieux de provenances sont donc beaucoup plus variées que précédemment, mais la mobilité, est elle-aussi, fortement accrue: nationalement, les enfants sont déplacés à l'intérieur du pays ; internationalement, les enfants continuent régulièrement leur trajet au-delà des frontières néerlandaises.

\section{Un contexte social modifié}

6 Ces circonstances affectent aussi le contexte social et il est d'autant plus important de prendre en compte des facteurs de complication tels que des parcours scolaires extrêmement hachés voire inexistants. Les jeunes apprenants ont fait des expériences de rencontres avec plusieurs cultures, des habitudes et normes différentes; ils ont vécu des transitions multiples entre les différents systèmes et langues de scolarisation et ils ont pour la plupart développé des compétences inégales dans ces diverses langues. Ces expériences influent sur les perceptions identitaires des apprenants et de leurs enseignants. Cet aspect est essentiel lorsque l'on s'interroge sur les conséquences didactiques en matière de médiation, d'une langue vers l'autre, au travers de normes et d'habitudes diverses. Élevés dans des environnements multilingues et multiculturels changeants, les enfants ne peuvent plus s'identifier à une langue et une culture, mais plutôt avec une gamme de langues et de cultures rencontrées dans des situations différentes. Cela peut être particulièrement pertinent pour les enfants réfugiés, puisque beaucoup ont connu une trajectoire ponctuée de séjours multiples dans différents pays avant d'arriver à leur destination actuelle. À cela s'ajoute les expériences traumatisantes de déplacement, voire de guerre ou de pauvreté économique et sociale. Dans le cadre de notre recherche, nous avons pu constater que plus l'enfant avait été exposé à des expériences traumatisantes graves et plus son comportement était jugé comme dysfonctionnant par les enseignants (Le Pichon- Vorstman, Baauw \& de Jonge, 2015). Cela suggère également que le plurilinguisme est devenu un mode de vie plutôt qu'un problème à résoudre (voir par exemple Creese et Blackledge, 2010 ; Busch, 2012). 


\section{Le contexte européen de mobilité}

7 Bien qu'elles aient rapidement gagné du terrain au cours de la dernière décennie, les motivations politiques et économiques ne sont pas les seules raisons qui expliquent l'hyper-mobilité des apprenants. Les étudiants sont également devenus plus mobiles suite au succès du programme de bourses Erasmus et des programmes d'échange similaires. Ainsi, au court de leurs séjours, ils créent de nouveaux liens avec d'autres étudiants internationaux et leurs pratiques linguistiques peuvent être éloignées de la traditionnelle acquisition d'une langue dite «seconde " (Anquetil \& Molinié, 2011, Della Chiesa et al., 2012). Les enfants dont les parents se déplacent pour des raisons professionnelles ou les enfants de couples multinationaux en sont un autre exemple. Pour cette population, les écoles européennes et internationales dispensent une éducation plurilingue et pluriculturelle qui leur permet de se déplacer dans toute l'Europe (Vez, 2009), éducation qui leur garantit la continuité éducative du système scolaire. Mais, le nombre de ces écoles est largement insuffisant pour répondre à la demande, et l'éducation dans ces écoles ou dans ces programmes est souvent onéreuse, ce qui exclut un nombre certain d'élèves.

Ces exemples illustrent un aspect facilement négligé de l'éducation dite plurilingue dont l'accès dans la pratique est assez limité. C'est l'une des raisons pour lesquelles le plurilinguisme est officiellement promu par l'Union Européenne : plus les pays adoptent une approche plurilingue dans l'éducation, plus la mobilité entre les différents pays est facilitée. On comprend alors que la didactique du plurilinguisme est au cœur de la médiation pour la gestion de la diversité en milieu scolaire et que les enseignants de langue deviennent les médiateurs indispensables, clefs de voute d'un système scolaire fondé sur l'intégration homogénéisante à un système qui promeut l'inclusion de tous les apprenants. Et pourtant, dans la pratique, l'accès à ces systèmes est assez limité. Ce sont donc les systèmes éducatifs qui doivent s'adapter à ces réalités complexes sans sousestimer les risques encourus, à la fois en termes d'apprentissage et de perte de diversité linguistique et culturelle si l'on se contente d'une inclusion interculturelle normative (Herzog-Punzenberger, Le Pichon-Vorstman, Siarova, 2017).

\section{Didactique et médiation des langues et des cultures}

\section{Résistances à la médiation}

9 Ces nouveaux modèles migratoires entrainent pour l'éducation, une prise en compte des répertoires individuels des apprenants (Achugar et Carpenter, 2012) et nous amènent à recentrer les objectifs éducatifs sur les aspects plurilingues et multilatéraux (Auger, 2012). Les différents groupes d'apprenants mentionnés ci-dessus ont tous le potentiel de développer des atouts importants cognitifs et métacognitifs. L'exemple des résultats d'une recherche menée récemment aux Pays-Bas est exemplaire à plus d'un titre (Le Pichon- Vorstman, Baauw \& Vorstman, à paraître). Les Pays-Bas évaluent tous les apprenants de l'école primaire, à l'échelle nationale, dans un certain nombre de matières, plusieurs fois par an: mathématiques, vocabulaire, compréhension de textes, entre autres. Comparant les résultats à ces évaluations nationales d'un groupe d'apprenants nouvellement arrivés, 2, 3, et 4 ans après leur inclusion dans le système scolaire néerlandais, il est apparu que la moyenne des résultats des apprenants dits 
nouvellement arrivés était comparable à celle de leurs pairs et parfois même, dépassait la moyenne nationale.

On pourrait qualifier ces résultats de positifs. Pourtant, une deuxième analyse des données a montré que ces mêmes apprenants commençaient avec un an et demi de retard sur leurs pairs et, plus grave, que ce retard augmentait avec l'âge de manière significative. Les interprétations sont complexes : il s'agit pour cette recherche d'un petit groupe d'apprenants et ces résultats impliquent de nombreux facteurs intrinsèquement liés: les attentes et les objectifs des enseignants, la structure de l'enseignement, les histoires et parcours individuels des apprenants, etc. Tous ces facteurs ont leur responsabilité dans ce qui pourrait apparaître comme une tendance vers le décrochage scolaire. En effet, une enquête de terrain nous a montré qu'après la classe d'insertion, les enfants étaient effectivement presque systématiquement envoyés dans des niveaux inférieurs à leur âge, entrainant peut-être une perte de motivation de la part des élèves et une baisse de la stimulation cognitive et émotionnelle par l'enseignant, autrement dit, un manque en matière de médiation cognitive (stimulation du développement de la connaissance) et de médiation relationnelle (maintenir le lien) à travers l'activité langagière (Coste et Cavalli, 2015). Lorsqu'ils évoluent dans un nouvel environnement scolaire langagier, ces élèves ont besoin de soutien pour réussir à transférer leurs connaissances d'une langue à une autre. Ils ont également besoin de soutien pour apprendre comment communiquer avec succès - activement et passivement - et développer leurs compétences cognitives dans différentes matières à travers de nouvelles langues. Coste et Cavalli parlent de «mobilités facilitées et rendues fluides par l'œuvre de médiation d'acteurs divers à l'intérieur d'un cadre institutionnel» (2015: 36). Cela nécessite des approches d'enseignement et d'apprentissage des langues articulées qui, malheureusement, ne sont pas encore en place dans la majorité des institutions scolaires.

\section{Rôle de la médiation en didactique des langues et des cultures}

11 Lorsque les élèves sont scolarisés dans un système qui soutient leur plurilinguisme (O'Laoire, 2005 ; Le Pichon-Vorstman et al., 2009) et lorsque la langue est explicitement enseignée (Kim et al., 2015), les enfants plurilingues ont plus de chance de réussir leur parcours scolaire. Cummins, suivi par beaucoup d'autres chercheurs, a montré que le transfert positif de connaissances et de compétences à l'échelle des langues est renforcé par l'établissement de liens explicites entre les langues (Cummins, 1991). Tous montrent que la valorisation du contexte linguistique et culturel unique de chaque enfant favorise la réussite scolaire en stimulant la confiance en soi et l'estime de soi (voir par exemple Moore, 2006; Sierens et van Avermaet, 2013 ; Extra et Yagmur, 2012). En outre, plus le soutien au plurilinguisme individuel est intense et cohérent, plus les avantages scolaires sont palpables (Ball, 2011). L'inverse, soit la dévalorisation systématique des cultures et des langues des apprenants, ne peut avoir qu'un impact négatif sur leur réussite et leur motivation globale (voir par exemple Benson et Elorza, 2015). Ainsi, l'adoption d'un habitus multilingue dans la politique et la pratique éducatives peut également être une approche précieuse pour promouvoir la compétence communicative et favoriser le rendement scolaire (Benson, 2013).

12 Un aspect particulièrement difficile de la diversité dans l'éducation est qu'elle implique intrinsèquement un ensemble déséquilibré de compétences dans les différentes langues (Grosjean, 1989). Il serait tout à fait réducteur de ne parler que de langues. Lorsque les 
élèves se déplacent d'un endroit à un autre, ou d'une langue à l'autre, ils développent différents ensembles de connaissances dans leurs différentes langues, cultures. Ces langues et cultures ne sont plus ni étrangères ni secondes, chacune de ces qualifications représentant le statut didactique que nous leur donnons, elles font déjà partie du patrimoine de l'apprenant à un degré plus ou moins développé. Les apprenants dans leurs déplacements, se familiarisent avec la terre sur laquelle ils se déplacent, ils en puisent les ressources à condition toutefois que ces ressources soient disponibles et accessibles. On rencontre un certain nombre de témoignages d'enfants de couple multinationaux symboliquement rejetés des cours de langue dite étrangère qui ne sont en réalité que l'une de leurs langues maternelles. L'essentiel de la problématique que cette situation soulève et une problématique de pouvoir : au risque de paraphraser Léonard de $\mathrm{Vinci}^{1}$, lorsque l'apprenant dépasse le maitre, sont-ils encore légitimes, l'étudiant en tant qu'apprenant et le maitre en tant que maitre ? Ne peut-on pas enseigner cette légitimité à nos enseignants par le biais de la médiation?

Les résistances sont nombreuses et les apprenants particulièrement conscients des problématiques de pouvoirs qui sont impliquées dans l'enseignement, quel que soit leur âge et en particulier dans l'enseignement des langues et des cultures. Cette métacognition accrue que nous mettons souvent en avant pour vanter le plurilinguisme, ne s'arrête pas au contenu de ce qui est enseigné mais se prolonge dans la situation d'enseignement ellemême. Cependant, ces problématiques de pouvoir en didactique des langues aboutissent souvent à un rejet par l'apprenant de la langue dans le contexte de l'institution au moins et ne peuvent qu'affecter les identités en construction et fragiliser des apprenants et de leurs enseignants. Le concept « langue étrangère » représente donc un statut didactique exclusif qui, de fait, délégitime l'apprenant pour qui la langue en question n'est pas étrangère.

\section{Responsabilité en matière de médiation}

Cette situation soulève un autre problème: pourquoi partir du principe que les enseignants qui enseignent à des apprenants aux expériences linguistiques et culturelles extrêmement variées, devraient compter sur leurs propres ressources en matière de plurilinguisme pour mettre en place la médiation que ces situations nouvelles et complexes nécessitent? L'hypothèse implicite selon laquelle les enseignants disposent d'une expertise suffisante pour traiter le plurilinguisme des élèves n'est pas fondée sur la réalité. Il n'est pas surprenant que les enseignants se plaignent souvent de l'absence de soutien et d'une formation pertinente (Cummins et al., 2005). TALIS 2013 révèle par exemple que l'enseignement dans un contexte multiculturel et multilingue est l'un des cinq principaux domaines dans lesquels les enseignants manifestent le plus grand besoin de perfectionnement professionnel pour faire face aux déficits actuels (OCDE, 2014; Herzog-Punzenberger, Le Pichon-Vorstman, Siarova, 2017). En tout état de cause, l'arrivée récente de réfugiés a créé un réveil soudain dans de nombreuses régions d'Europe (et au-delà); des pays comme les Pays-Bas commencent à se rendre compte que leurs systèmes scolaires sont fortement orientés vers des élèves imaginés monolingues et prennent progressivement conscience d'un réel besoin de professionnalisation des enseignants en ce qui concerne le plurilinguisme et l'interculturel (Sierens \& van Avermaet, 2013, Le Pichon- Vorstman, 2012). 

nombreux autres pays, il pose des défis entièrement nouveaux au système éducatif et même à la société. Il n'est donc pas surprenant que les pays européens diffèrent grandement en fonction de leurs capacités à faire face aux demandes croissantes du contexte éducatif. La Frise, région du nord des Pays-Bas, région dotée d'une minorité historique où les habitants se sont battus pendant des années pour obtenir la reconnaissance de leur langue, a par exemple, adopté des stratégies plurilingues afin d'accroître la valeur et le respect de la langue et de la culture frisonne. La directrice d'une école primaire bilingue proche d'un camp de réfugiés, expliquait qu'elle recevait de plus en plus d'enfants venus d'ailleurs. Le conseil de l'école s'est donc réuni d'urgence pour établir une politique linguistique qui écarterait les langues de ces enfants de l'école afin de protéger le bilinguisme visé. Pourtant, le conseil a rapidement réalisé qu'il était en train d'exclure les langues des enfants alors que ses membres s'étaient battus pour imposer la leur. Le plurilinguisme initial et leur propre combat, leur ont permis cette prise de conscience. Ils sont alors entrés dans un projet incluant des stratégies d'interlangue. Depuis, les parents viennent à l'école, lire dans leurs langues aux enfants et les enfants apprennent les langues des autres en cours et pendant la récréation. Les médiateurs sont donc nombreux: ce sont les enseignants (de Néerlandais et Frison langues secondes), les parents, les enfants eux-mêmes. Ce récit nous montre que la médiation est contagieuse et rejoint les propos de Garcia lorsqu'elle dit que « les individus ne possèdent pas une langue ou une autre, ils font de la langue $»^{2}$ (Garcia, 2014, p. 149). Le médiateur est alors créateur de cohésion. Cette ouverture à la diversité est inscrite dans les patrimoines langagiers et culturels les plus diversifiés. Dans ces situations, une réflexion poussée sur les résistances personnelles, les compétences interculturelles et une didactique du plurilinguisme, peuvent suffire à faire entrer les acteurs de l'éducation dans une démarche interculturelle de médiation.

\section{Conclusion}

17 Dans l'ensemble, le défi politique est formidable et des recherches supplémentaires sont nécessaires pour fournir des recommandations fondées sur des données probantes qui puissent aider les décideurs de l'éducation à trouver des moyens pour relever les défis et opportunités que le plurilinguisme des apprenants apporte aux systèmes éducatifs en Europe et au-delà (Herzog-Punzenberger, Le Pichon-Vorstman \& Siarova, 2017). Pourtant, même dans des contextes où le multilinguisme est la norme, les salles de classe avec des apprenants très diversifiés sont encore perçues comme un défi qui n'est pas toujours abordé de manière adéquate, comme le montrent à la fois la séparation encore trop stricte des langues dans l'institution scolaire et académique ou l'évaluation souvent exclusivement abordée dans la langue dominante (Breton-Carbonneau et al., 2012). Le profond changement sociétal provoqué par les nouveaux schémas migratoires a créé le besoin de repenser le cadre de compétences-clés pour l'apprentissage tout au long de la 
vie au XXIe siècle et les approches d'enseignement des langues dans les écoles. Les notions de communication dans la (les) langue (s) maternelle (s), la (les) langue (s) de scolarité, les langues supplémentaires et la communication dans des langues autres que celles utilisées à l'école font de plus en plus l'objet de discussions. Le cadre de compétences actuel, qui vise à accroître la citoyenneté active, semble quelque peu biaisé, comme en témoigne l'utilisation de concepts qui reflètent une approche monolingue de l'éducation. Ainsi, les notions de "langue maternelle», de "bilinguisme ", de «langue étrangère » ou d' " acquisition de la langue seconde » réfèrent implicitement à un ordre fixe d'acquisition des langues, à une hiérarchie des valeurs et des usages et donc à une tendance monolingue (May, 2013, Auer, 2007, Gogolin, 1997). Ces concepts se réfèrent à des espaces géographiques bien définis. Pourtant, ce sont précisément ces espaces qui se transforment par une mobilité accrue et une diversité croissante. L'étude des paysages linguistiques montre que les frontières entre les langues sont poreuses (Zarate et al., 2011). Les espaces de langue et de culture précédemment confinés sont en transition vers cet amalgame de langues et de cultures. Ce changement majeur nécessite une plus grande implication en termes de médiation et de compréhension interculturelle et un besoin de « réflexion» (Byrd-Clark \& Dervin, $2014: 1$ ) pour identifier les besoins de médiations en vue de l'inclusion de tous les élèves. Il s'en suit qu'afin d'améliorer l'éducation dans des contextes de plus en plus diversifiés, des stratégies médiatrices et des ressources efficaces sont nécessaires dans les écoles à travers l'Europe.

Dans cette perspective, il est important de noter que la politique multilingue de la Commission Européenne a été interprétée par la plupart des systèmes éducatifs comme une incitation à introduire l'apprentissage formel des langues par des cours de langue traditionnels. Compte tenu de la situation actuelle, cette politique devrait être repensée en termes d'habitus plurilingue (comme le discutent Benson \& Elorza, 2015), chaque langue de la biographie linguistique d'un individu étant également louée et valorisée.

19 En résumé, l'émergence de nouvelles formes de biographies langagières, stimulées par la modification des modes de migration et l'évolution des objectifs éducatifs et professionnels, met en question les approches traditionnelles de l'apprentissage et de l'enseignement des langues. Elle remet aussi en question les problématiques de niveau de langue et d'évaluation, qui portent atteinte au rôle traditionnel de l'enseignant, et en particulier de l'enseignant de langue, comme unique détenteur de la connaissance. Si l'on comprend le concept de médiation, dans son sens juridique large, selon les mots de Pruvost, comme celui d'une «entremise destinée à rendre possible une conciliation ou une réconciliation entre des personnes, des partis» (2016:9), il me semble que la didactique des langues peut jouer un rôle conciliateur entre l' institution scolaire en déclin, monolingue et monoculturelle et l' institution scolaire nouvelle, composée d'acteurs plurilingues et pluriculturels à condition que les acteurs, c'est à dire les enseignants, soient formés à la médiation des langues et des cultures. 


\section{BIBLIOGRAPHIE}

Achugar, M. ; Carpenter, B. (2002). Developing disciplinary literacy in a multilingual history classroom, Linguistics and Education, vol. 23, pp. 262- 276.

Anquetil, M. ; Molinié, M. (2001). The Erasmus experience from a reflexive perspective ; considering and constructing social actors'. In : Zarate, G. ; Lévy, D. ; Kramsh, C. (eds.) Handbook of Multilingualism and multiculturalism, pp. 77-81.

Auer, P. (2007). Monolingual bias in bilingualism research - or: Why bilingual talk is (still) a challenge for linguistics. In: M. Heller (ed.), Bilingualism: A social approach. Houndmills : Palgrave, pp. 319-339.

Auger, N. (2012). Favoriser les approches interculturelles et interlinguistiques : comment tirer parti des répertoires plurilingues et pluriculturelles des élèves? In. Le français comme langue de scolarisation, scérén CNDP-CRDP, pp. 168.

Ball, J. (2011). Enhancing learning of children from diverse language backgrounds: Mother tongue-based bilingual or multilingual education in the early years, Analytical review commissioned by UNESCO Education centre.

Benson, C ; Elorza, I. (2015). Multilingual education for all (MEFA) : Empowering non-dominant languages and cultures through multilingual curricular development. In. The Sage handbook of curriculum, pedagogy and assessment : Two volume set, pp. 557-574.

Blommaert, J. (2010). The sociolinguistics of globalization. Cambridge : Cambridge University Press.

Breton-Carbonneau, G. ; Cleghorn, A. ; Evans, R. ; Pesco, D. (2012). Pedagogical and political encounters in linguistically and culturally diverse primary classrooms : examples from Quebec, Canada, and Gauteng, South Africa', Compare: A Journal of Comparative and International Education, vol. 42, n 3, pp. 373-391.

Busch, B. (2012). The Linguistic repertoire revisited. Applied Linguistics, pp. 1-22.

Byrd-Clark, J.; Dervin, F. (2014). Reflexivity in language and intercultural education. New York: Routledge.

Byrd-Clark, J. ; Dervin, F. (2014). Reflexivity in language and intercultural education, New York: Routledge.

Coste, D. et Cavalli, M. (2015). Education, mobilité, altérité Les fonctions de médiation de l'école. Conseil de l'Europe.

Creese, A ; Blackledge, A. (2010). Translanguaging in the bilingual classroom : a pedagogy for learning and teaching? The Modern Language Journal, vol. 94, $\mathrm{n}^{\circ}$ 1, 103-115.

Cummins, J. (1991). Interdependence of first- and second-language proficiency in bilingual children'. In : Bialystok, E. (ed), Language Processing in Bilingual Children. Cambridge : Cambridge University Press, pp. 70-89.

Cummins, J. ; Bismilla, V. ; Chow, P. ; Cohen, S. ; Giampapa, F. ; Leoni, L. ; Sandhu, P. ; Sastri, P. (2005). Affirming Identity in multilingual classrooms by welcoming a student's home language into the classroom, schools actively engage English language learners in literacy, Educational leadership, vol. 63, $\mathrm{n}^{\circ} 1$, pp. 38-43. 
Della Chiesa, B., Scott, J. ; Hinton, C. (eds.). (2012). Languages in a Global World : Learning for Better Cultural Understanding, OECD Publishing, Paris.

European Commission. (2003). Promoting language learning and linguistic diversity: An action plan 2004-2006. Luxembourg : Office for Official Publications of the European Communities. ISBN 92-894-6626-X

European Commission. (2012). Study on Educational Support for Newly Arrived Migrant Children. ISBN 978-92-79-27950-8

Extra, G. ; Yagmur, K. (eds.) (2012). Language Rich Europe: Trends in Policies and Practices for multilingualism in Europe, Cambridge University Press, on behalf of the British Council.

García, O. (2014). Becoming bilingual and biliterate : Sociolinguistic and sociopolitical considerations. In C. Addison Stone, E. R. Silliman, B. J. Ehren, \& G. P. Wallach (eds.), Handbook of language and literacy: Development and disorders, pp. 145-160. New York: The Guilford Press.

Gogolin, I. (1997). The 'monolingual habitus' as the Common Feature in Teaching in the Language of the Majority in Different Countries. Per Linguam vol. 13 n.2, pp. 38-49.

Grosjean, F. (1989). Neurolinguists, beware! The bilingual is not two monolinguals in one person. Brain and Language, vol. 36, pp. 3-15.

Herzog-Punzenberger, B. ; Le Pichon-Vorstman, E. ; Siarova, H. (2017). Multilingual Education in the Light of Diversity : Lessons Learned, NESET II report, Luxembourg : Publications Office of the European Union, 2017. doi : 10.2766/71255.

Hornberger, N. H. (2004). The Continua of Biliteracy and the Bilingual Educator: Educational Linguistics in Practice, International Journal of Bilingual Education and Bilingualism, vol. 7, $\mathrm{n}^{\circ} 2-3$, pp. 155-171.

Hornberger, N. H. ; Link, H. (2012). Translanguaging and transnational literacies in multilingual classrooms : a biliteracy lens, International Journal of Bilingual Education and Bilingualism, vol. 15, pp. 261-278.

Kim, T.J. ; Kuo, L-J. ; Ramirez, G ; Wu, S., Ku, Y-M. ; de Marin, S. ; Ball, A. ; Eslami, Z. (2015). The relationship be-tween bilingual experience and the development of morphological and morphosyntactic awareness : a cross-linguistic study of classroom discourse, Language Awareness, vol. 24, n.4, pp. 332-354.

Le Pichon- Vorstman, E. (2012). Transitions et plurilinguisme : L'évaluation des jeunes enfants alloglottes issus des minorités linguistiques aux Pays-Bas et en Suède, à l'école publique. http:// admee2012.uni.lu/pdf2012/A26_01.pdf

Le Pichon- Vorstman, E., van Erning, R., Baauw, S. (2015). Country report : The Netherlands. Education of Newly arrive migrant pupils, https://edinaplatform.eu/

Le Pichon- Vorstman, E. ; Baauw, S ; Vorstman, J.A.S. (À paraitre). School development of Newly arrived migrant pupils in The Netherlands at primary school level.

Le Pichon-Vorstman, E. Baauw, S. (2014). Onderzoeksrapport : Taalschool Utrecht. Unpublished report.

Le Pichon-Vorstman, E. ; de Swart, H. ; Ceginskas, V. ; van den Bergh, H. (2009). Language Learning Experience in school context and metacognitive awareness of multilingual children'. International Journal of Multilingualism, vol. 6, n. 3, pp. 256-280.

May, S. (ed.). (2013). The Multilingual Turn: Implications for SLA, TESOL, and bilingual education, London and New York : Routledge, pp. 240. 
$\mathrm{O}^{\prime}$ Laoire, M. (2005). Three languages in the schools in Ireland. International Journal of the Sociology of Language, pp. 171.

OECD. (2014). TALIS 2013 Results : An International Perspective on Teaching and Learning, OECD Publishing. http://www.oecd.org/edu/school/talis-2013-results.htm

Pruvost, J. (2016). « Du « moyenneur » ou « médiateur » à la « médiation », sous ses diverses facettes lexicographiques », Ela. Études de linguistique appliquée, vol. 1, n. 181, pp. 5-10.

Sierens, S. ; van Avermaet, P. (2013). Language diversity in education : evolving from multilingual education to functional multilingual learning. In : Little, D. ; Leung, C. ; Van Avermaet, P., Managing diversity in Education : languages, policies and pedagogies, pp. 204-219. Multilingual Matters. Bristol, Buffalo, Toronto.

Vertovec, S. (2007). Super-diversity and its implications. Ethnic and Racial Studies, vol. 30 n. 6, pp. 1024-1054.

Vez, J-M. (2009). Multilingual Education in Europe : Policy Developments, Porta Linguarum, pp. 7-24.

Zarate, G. ; Lévy, D. ; Kramsh, C. (2011). Handbook of Multilingualism and multiculturalism. Editions des Archives Contemporaines, pp. 417.

\section{NOTES}

1. "Tristo è quel discepolo che non avanza il suo maestro." (Annexe 7.3 : Codex Forster III, 66 vo.)

2. Notre traduction: "people are involved in languaging"

\section{RÉSUMÉS}

L'aspect de continuité dans l'enseignement est très fortement remis en cause par les mouvements de migration actuels. Enrichissement ou appauvrissement? La diversité doit-elle conduire à un inéluctable nivellement par le bas ou au contraire, peut-elle jouer le rôle de renouvellement éducatif et d'explosion de la connaissance? Quel rôle médiateur peut ou doit jouer la didactique des langues afin de contrebalancer l'effet migratoire sur la continuité du développement de l'enfant/ étudiant apprenant dans un système scolaire fossilisé ? Qu'en est-il de l'enseignant-apprenant? Quelles leçons peut-on tirer des expériences passées et dans un contexte européen? Dans cet article, j'insiste sur une vision inclusive et contextualisée de l'éducation. Je donne la parole aux apprenants et aux enseignants que j'ai rencontrés pour qu'ils nous parlent de leurs pratiques, de leurs savoirs, de leurs croyances sur l'enseignement. J'adopte une approche interdisciplinaire intégrée de l'enseignement des langues seconde, étrangère, de l'enseignement bilingue. À la suite de Hornberger, je plaide pour que l'éducation, la recherche et la théorie deviennent intégralement liées et mutuellement informées (Hornberger, 2004) au service de la médiation des langues et des cultures.

The aspect of continuity in education is very strongly challenged by the current movements of migration. Enrichment or impoverishment? Should diversity lead to an inevitable leveling from 
the bottom or, on the contrary, can it play the role of educational renewal and the explosion of knowledge? What mediating role can or should language teaching play to offset the migratory effect on the continuity of development of the child / student learning in a fossilized school system ? What about the teacher-learner? What lessons can be learned from past experiences and in a European context? In this article, I insist on an inclusive and contextualized view of education. I give the floor to the learners and teachers I have met to tell us about their practices, their knowledge and their beliefs about teaching. I adopt an integrated interdisciplinary approach to teaching second-, second-, and foreign languages in bilingual education. Following Hornberger, I advocate that education, research and theory become fully interrelated and mutually informed (Hornberger, 2004) in service of the mediation of languages and cultures.

\section{INDEX}

Mots-clés : médiation, migration, didactique des langues étrangères et secondes, mobilité, plurilinguisme, éducation

Keywords : mediation, migration, foreign and second language education, mobility, plurilingualism, education

\section{AUTEUR}

\section{EMMANUELLE LE PICHON-VORSTMAN}

Université de Toronto

Dr. Emmanuelle Le Pichon-Vorstman est professeure adjointe à l'Université de Toronto, OISE. Auparavant, elle a travaillé pendant 10 ans dans le Département de langues, littérature et communication de l'université d'Utrecht aux Pays-Bas. Ses recherches portent sur le plurilinguisme en éducation. Depuis 2009, elle a dirigé plusieurs projets nationaux et internationaux sur l'inclusion dans l'éducation des élèves issus des minorités. Emmanuelle Le Pichon-Vorstman a travaillé en tant que consultant, chercheur, évaluateur et examinateur pour plusieurs organisations et revues internationales. Elle a participé à des analyses politiques, notamment pour la Commission Européenne (NESET II, Sirius, Erasmus +) et le Migration Policy Institute (Washington DC, mai 2015). Son vif intérêt pour les politiques migratoires l'a amenée à mener des recherches sur des questions liées à l'éducation plurilingue, en particulier sur l'insertion dans l'éducation des apprenants nouvellement arrivés en Europe (projet TRAM, projet Taalschool, EDINA) et des apprenants autochtones au Suriname (en collaboration avec la Fondation Rutu). En 2015, son rapport de recherche a été au centre d'un débat au parlement néerlandais qui a conduit à l'obtention d'une deuxième année de financement en faveur des apprenants refugiés aux Pays-Bas. Au cours de ces dernières années, elle a siégé, entre autres, aux conseils d'administration de l'Association néerlandaise de linguistique appliquée (2012-2017) et du Comité d'éthique de l'Institut de linguistique d'Utrecht (2016-2017) 